\title{
DeViCE-To-Device (D2D) COMMUNiCATION UNDER LTE-ADVANCED NETWORKS
}

\author{
Magri Hicham ${ }^{1}$, Noreddine Abghour ${ }^{2}$ and Mohammed Ouzzif ${ }^{1}$ \\ ${ }^{1}$ RITM Research Lab,ESTC , Hassan II University ,Casablanca, Morocco \\ ${ }^{2}$ FSAC, Hassan II University,Casablanca, Morocco
}

\begin{abstract}
Device-to-Device (D2D) communication is a new technology that offer many advantages for the LTEadvanced network such us wireless peer-to-peer services and higher spectral efficiency. It is also considered as one of promising techniques for the $5 G$ wireless communications system and used in so many different fields such as network traffic offloading, public safety, social services and applications such as gaming and military applications. The goal of this paper is to present advances on the current 3GPP LTE-advanced system related to Device-to-Device (D2D). In this paper, we provide an overview of the $D 2 D$ types based on the communication spectrum of $D 2 D$ transmission, namely Inband D2D communication and Outband D2D communication. Then we present the advantages and disadvantages of each D2D mode. Moreover, architecture and protocol enhancements for D2D communications under LTE-A network are described.
\end{abstract}

\section{KEYWORDS}

D2D;LTE-advanced;Inband D2D;Outband D2D;3GPP;5G.

\section{INTRODUCTION}

In recent years, exponential growth of wireless communication and data traffic, high demands for broadband mobile wireless communications and the emergence of new wireless multimedia applications and services are constituted the key drivers to the development of the Long Term Evolution-Advanced (LTE-A) network.

One of the main challenges of LTE-advanced is to recover the local-area services and enhance spectrum efficiency.To achieve those goals technical capabilities are required. Device to Device (Device to Device (D2D) communications is new technology that offer wireless peer-to-peer services and improve spectrum utilisation in LTE-advanced network [1][2]. D2D communications was initially proposed in cellular network as a new paradigm to enhance network performance.

The motivation for D2D come directly from the user requirements and D2D communications will serve specific future needs. These needs include new types of short range services and data intensive short range applications[3]. The emergence of context-aware and multimedia applications have constituted the motivation of using D2D technology.D2D communications will allow new types of services such multimedia downloading, video streaming, online gaming and peer-to-peer (P2P) file sharing.

DOI : 10.5121/ijwmn.2016.8102 
International Journal of Wireless \& Mobile Networks (IJWMN) Vol. 8, No. 1, February 2016

D2D communication refers to technology that enable the communication between multiple D2D devices or users without having base station or intermediary devices on a network [4] . This makes D2D communication a key technology to solve some problems such as coverage and interference management .It increase also spectrum utilisation and capacity and enhance network performance and throughput .Differing from the Bluetooth and WiFi-direct, we categorize the D2D communication in cellular network based on the spectrum in which D2D communications occurs. Thus, D2D communication can occur on cellular system and in this case called Inband D2D or can occur in unlicensed spectrum and is called Outband D2D. The motivation of choosing inband communications is usually the high control over licensed spectrum.

In D2D communications the cellular network can handle phone calls and internet data traffic without additional networks load from the promotional material. However, there are many complexities of setting up and to deploy D2D communications in LTE advanced networks. These challenges and complexities include:

- D2D devices cause interference to the cellular users which affect the performance of the network devices.

- D2D communications define new QoS requirements that must be addressed.

Hence, LTE-advanced present two techniques of D2D communications that use Session

Initiation Protocol (SIP) and Internet Protocol (IP).These techniques have the benefit of providing the control over the D2D connectivity to the operator. The integration of D2D communications in LTE-A must take into account LTE-A interfaces and network elements.

While the need for physical layer backward capability imposes the D2D devices to utilize for their links the current structure of the spectrum resources [5].

\section{Classification Of D2D Communication}

D2D communication in cellular network can be categorized into both Inband D2D and Outband D2D based on the spectrum in which D2D communications occurs [6]. D2D communications is divided into two modes or categories called ' Inband underlay mode ' when the D2D communications use the cellular resources and spectrum and ' Inband overlay mode 'when cellular resources are allocated for the two D2D end devices that communicate directly (eg, Figure.1) .

High control over licensed spectrum is the key motivating factor for choosing the Inband D2D communication. In other hand, the main motivation of using Outband D2D communications is the capacity to eliminate the interference between D2D links.Furthermore, Outband D2D communications is faced with a lot of challenges in the coordination between different bands.

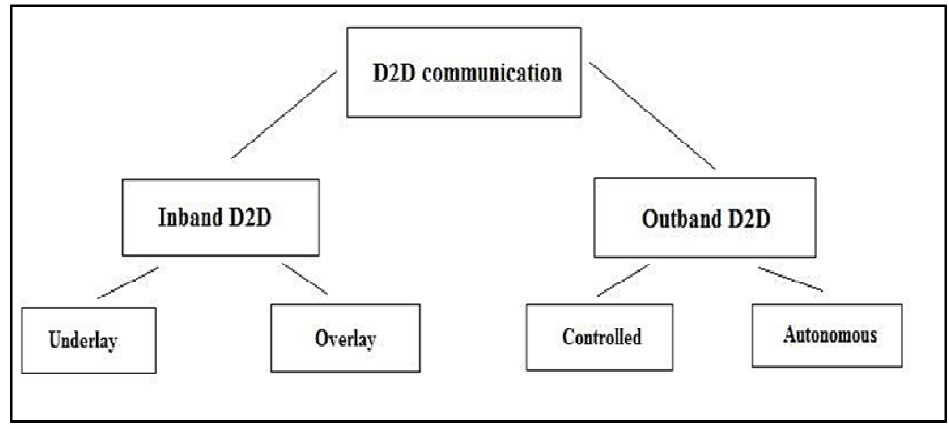

Figure.1 .Classification of D2D communication

In this section, we provide a formal definition for each category of D2D communication and an overview to advantages and disadvantages of each D2D mode. 
International Journal of Wireless \& Mobile Networks (IJWMN) Vol. 8, No. 1, February 2016

\subsection{Inband Communication}

The motivation for choosing inband communication is the high control over licensed spectrum. [2][3][7], Results show that QoS provisioning had lot of requirements by the consideration of the uncontrollability of interference in the unlicensed spectrum . In [3],the feasibility of D2D communications and its impacts in licensed spectrum are studied by simulation and analysis of different scenarios and authors show that by tolerating the increase of interference in licensed spectrum the D2D communication will be possible .

To improve the spectrum efficiency the D2D inband can reuse the time and frequency ressources by d2D users (i.e Underlay) or allocating time and frequency resources occupied by D2D users (i.e overlay) [8]. Under this, Inband communications can be divided into underlay and overlay categories.

The most disadvantage of inband $\mathrm{D} 2 \mathrm{D}$ is the interference caused by $\mathrm{D} 2 \mathrm{D}$ users to cellular communications. This interference can be mitigated by introducing high complexity resource allocation methods, which increase the computational overhead of the eNB of D2D users.

\subsubsection{Undelay Inband D2D mode}

In underlay inband mode, cellular and D2D communications share the same radio resources. To date, most of the papers in literature are dedicated to D2D communications underlaying cellular network [9] [10] [11].

Underlay inband can improve and enhance the performance of different targets such as spectrum efficiency, energy efficiency,and cellular coverage by the use of different techniques including diversity techniques,interference reduction ,resource allocation and by using nework coding [12][13][14][15],[16],[17].The papers, [14], [18] and [19] adopt more advanced mathematical techniques than the others.

The [20][21] works define and propose new algorithms and interference management strategy to achieve overall capacity enhancement and to solve mode selection problem in cellular networks and D2D systems. Another method is proposed in [22]. The computational complexity of the above mentioned method is higher but it is better than those proposed in [20][21].

To improve the system performance of D2D cellular system with QoS / power constraints, advanced techniques are required. These techniques include stochastic optimization, nonlinear programming, and integer optimization [23][24].

Several research papers have an interest in resolving interference problems in D2D communication underlaying cellular networks [25][26][29].In [26] paper and using numerical results authors discuss different problems sush as interference management, Optimization of Transmission Power and throughput maximization of D2D communications in the case of the coexistence of many D2D users and one cellular user. An interference avoidance scheme and system model is proposed in [27]. This scheme is based on overhearing the received signal power of cellular UEs .New management method is presented in [28] to enhance the overall capacity of cellular networks and device-to-device (D2D) systems. The integration of D2D communication in a LTE-Advanced network is discussed in [29]. In the same way, authors propose different mechanism such as D2D communication session setup and management procedures. Another scheme is given in [25] to manage the interference between D2D communications and cellular network. In [30], authors propose a network controlled algorithm with low computation complexity to efficiently maximize the reuse of cellular network spectrum and to minimize system capacity.

Finally, by allowing underlying direct D2D communications, LTE-advanced mobile network can offers several advantages such as low end-to-end latency and high spectral efficiency. 
International Journal of Wireless \& Mobile Networks (IJWMN) Vol. 8, No. 1, February 2016

\subsubsection{Overlay Inband D2D mode}

In this mode, cellular and D2D are given dedicated cellular resources and those cellular resources are subtracted from cellular users in order to eliminate interference for the D2D communications on cellular transmissions [31][32][33]. In [33], a BS-assisted scheduling and D2D power control was proposed in order to reduce D2D interference. The authors of [31] and [32] focus on relaying use-case of D2D. Specifically, [32] proposes to use the BS as a backup transmitter for D2D transmission and [31] use multiple D2D users as relays for multicasting. The performance of the proposed methods and algorithms in [31] and [32] are given under the low complexity which makes them practical for real scenarios. On the other side, propose a network controlled algorithm to maximize the cellular network spectrum reuse and to provide high performance for D2D users .

\subsection{Outband D2D communication}

Nowadays, Outband D2D communication is attracting the attention of many researchers .In this category, D2D communication is performed in the unlicensed spectrum such as ISM $2.4 \mathrm{G}$ which made the interference between D2D and cellular communications impossible. On the other hand, Outband D2D my suffer from the uncontrolled nature of unlicensed spectrum. To exploit the unlicensed spectrum it is necessary to have another extra interface that implements WIFIDirect ,Zig Bee or Bluetooth[34][35][36].

The coordination between two different bands for achieving Outband D2D communication has a lot of challenges because in most cases the D2D communications occur in the above mentioned extra interface [8]. Outband D2D communication can be classified into two categories or modes depend on the occurrence of the second interface. These modes are called controlled mode when the second interface in under cellular network or autonomous when D2D control is done by users and the occurrence of the second interface is not under cellular network. There are also several literatures that refer to Outband D2D communications. In [37][38] works ,energy efficiency and throughput performance are boosted by the use of gaming theory and clustering. The works in [39][23][40] and [41] aim to improve the performance of content distribution. Forthermore, authors of the [41] paper provide an evaluation of QoS parameters such as delay and traffic load. Simulation results shows that the D2D architecture proposed and simulated in this work provide delay and traffic load performance enhancement .

\subsubsection{D2D Outband communications: Controlled mode}

In this category of $\mathrm{D} 2 \mathrm{D}$ communications, all the literature propose to use the cellular network advanced management features to control D2D communication in order to improve the efficiency and reliability of D2D communications and to improve also the system performance in terms of throughput, power efficiency and multicast.

The authors of [42] and [43] papers discuss how D2D communications can be one of the key issues of the improvement of energy efficiency and throughput. They propose a novel scheme based on users cooperation and provide an analytical model to evaluate throughput and power consumption for the proposed scheme.

The work in [41] introduces a protocol that focuses on D2D communications using LTE and Wifi-Direct. The authors elaborate on required modification for messaging and signalling procedures of LTE and WiFi Direct technologies. The D2D communication paradigm can be implemented on LTE infrastructure by using the Wifi Direct and without making a major change in LTE protocols . 
International Journal of Wireless \& Mobile Networks (IJWMN) Vol. 8, No. 1, February 2016

\subsubsection{D2D Outband communications: Autonomous mode}

Nowadays, there are very few works on this category. Autonomous D2D communication is motivated by reducing the overhead of cellular network. This category does not require any changes at the BS (eNB) and can be deployed easily. The authors of [41] assume that the BS (eNB) does not always have traffic to send to all users at any time. They depict a scenario with two users, $\mathrm{u} 1$ and $\mathrm{u} 2$ that being served by one BS. They formulated the problem of choosing the optimal dispatching policy as a Markov decision process and studied is properties. Simulation results showed in [41] demonstrated that by considering the above mentioned approach delay performance can be improved and the major performance requirements is to increase power expenditure .

\subsection{Advantages and disadvantages of the different types of D2D communications}

Nowadays, Outband communications is attracting more and more attention. With the arrival of new smartphones and new mobile devices in phone market, device will be capable to implement Outband D2D schemes. Some researchers provides an overview of the advantages and disadvantages of Outband D2D communications. They are putting high hopes and are seeing it as the alternative to inbnd $\mathrm{D} 2 \mathrm{D}$ communication category .

A brief look of advantages and disadvantages of of Inband D2D and Outband D2D communications are given in the following tables :

Table 1. Advantages and disadvantages of Inband D2D communication

\begin{tabular}{|l|l|l|}
\hline \multicolumn{2}{|c|}{ Advantages of Inband D2D } & \multicolumn{3}{|c|}{ Disadvantages of Inband D2D } \\
\hline $\begin{array}{l}\text { Underlay D2D increases the spectral } \\
\text { efficiency of cellular spectrum by exploiting } \\
\text { the spatial diversity. }\end{array}$ & $\bullet \begin{array}{l}\text { Challenging control of level of } \\
\text { interference. } \\
\text { No possibility for D2D and cellular } \\
\text { Qimultaneous transmission. } \\
\text { entirely controlled by eNBs . } \\
\text { The possibility of using Inband D2D } \\
\text { communication on any mobile equipement . }\end{array}$ & $\begin{array}{l}\text { High complication of resource } \\
\text { allocation procedure and power } \\
\text { control }\end{array}$ \\
\hline
\end{tabular}

Table 2. Advantages and disadvantages of Outband D2D communication

\begin{tabular}{|c|c|}
\hline Advantages of Outband D2D & Disadvantages of Outband D2D \\
\hline $\begin{array}{l}\text { - } \text { Easier resource allocation. } \\
\text { - Possibility of simultaneous occurrence of } \\
\text { D2D and cellular users. } \\
\text { - None interference between D2D and } \\
\text { cellular subscribers. } \\
\text { - None necessary to devote cellular } \\
\text { resources to D2D spectrum }\end{array}$ & $\begin{array}{l}\text { - Necessary to decode and to encode } \\
\text { packets } \\
\text { D2D communication only used by } \\
\text { LTE and WiFi radio interfaces } \\
\text { - Need of the efficient power } \\
\text { management }\end{array}$ \\
\hline
\end{tabular}


International Journal of Wireless \& Mobile Networks (IJWMN) Vol. 8, No. 1, February 2016

\section{D2D Architecture Model AND Requirements In LTE-A NETWORKS}

LTE-advanced technology was the first platform that implements D2D communications. In June 2012, 3GPP was started a workshop about LTE Release 12 standardization that include new steps for D2D communication scenario and use to to introduce new services in LTEA[53].Integrity and high standards of D2D communications such as LTE Proximity service (ProSe) was discussed at the RAN 58 meeting [44][45] in Demeber 2012.

D2D use case and D2D communication requirements are given in [45] paper .An overview of D2D Proximity Services standardization in 3GPP LTE is given on workshop presented by Michael Gundlach at the European Conference on Networks and Communications (EUCNC) on June 2014.

[46] discussed the scenarios and requirements for D2D discovery and provided several considerations on discovery issues. Proposals about scenarios and requirements are presented. The D2D/ProSe functionality are in the first integrated in LTE Release 12 .Literature about ProSe is divided into two parts, called D2D discovery and D2D communication.

With proximity discovery or D2D discovery, users can discover other users that are in the proximity. Using E-UTRA, this process identifies that a UE is in proximity of another [45].

The D2D communication can be directlty between UEs by the use of the proximity discovery to reduce the need for manual interaction or via eNBs.

The direct communication between UEs can be also etablish without using proximity discovery function.In cellular network, direct communication is integrated with a cellular network and is naturally preceded by network supported proximity discovery.

The 3GPP system enablers for ProSe consists of the following functionalities :

- EPC-level ProSe Discovery

- ProSe direct discovery models.

- ProSe direct communication Modes.

- UE-to-Network relay or UE-to-UE relay.

Architectural enhancements for the EPC core network are necessery in order to facilitate D2D and cellular inks coexistence and for best D2D integration in LTE-A mobile networks .

An overview of architectural and protocol enhancements for the D2D integration in LTEAdvanced system is given in [48]. The paper describe also procedures and services used by D2D devices to discover each other . 
International Journal of Wireless \& Mobile Networks (IJWMN) Vol. 8, No. 1, February 2016

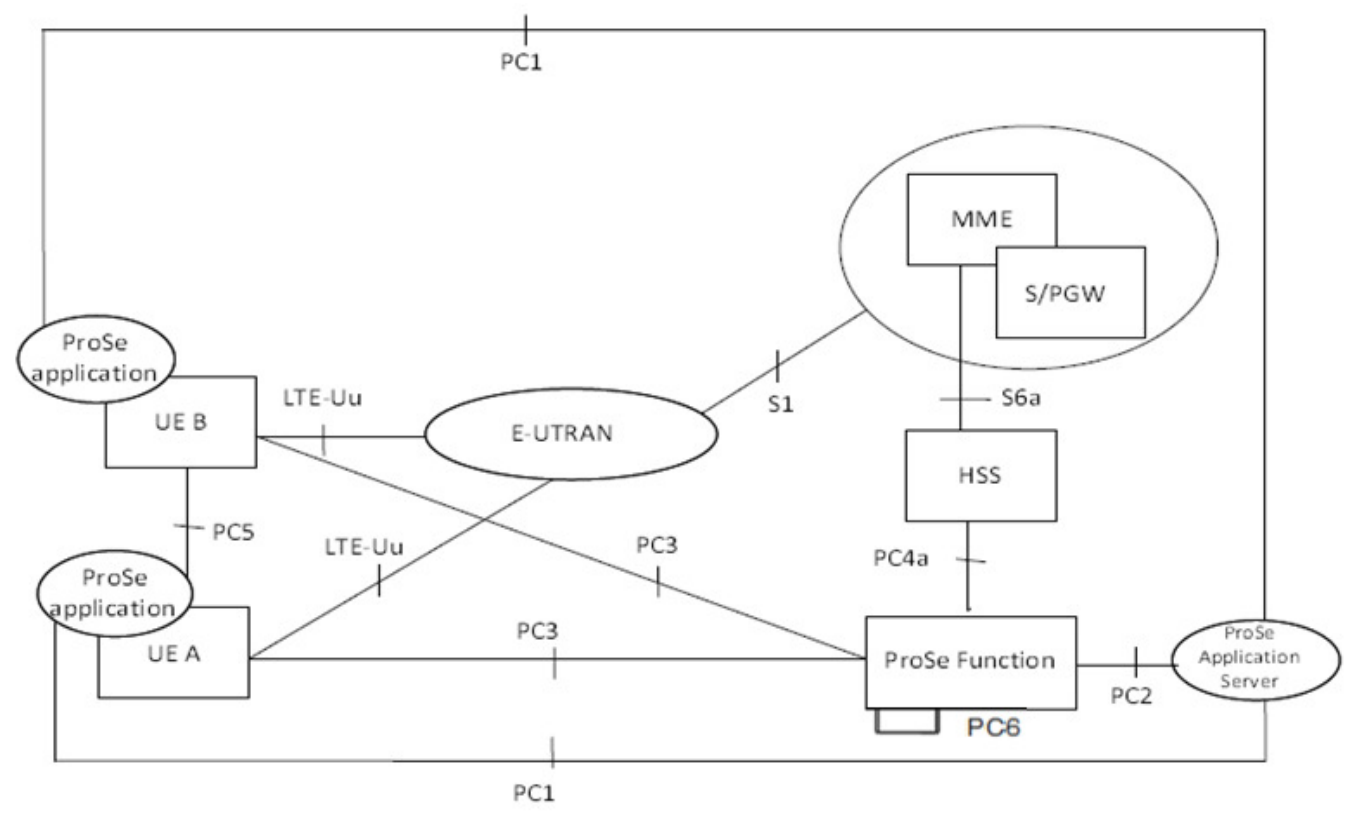

Figure 2. D2D communication architecture under LTE-A network

As depicted in Figure 2., The D2D system architecture under LTE-A consists of a number of new entities that are required for D2D communication. These entities are as follows:

ProSe App Server: It implements the ProSe capability for building the application functionality.It is responsible to determinate if the registration of UEs can be accepted or not and to activate ProSe actions such as ProSe discovery for a specific application. The ProSe application in the UE (ProSe) communicates with the ProSe Application Server via the application layer reference point PC1 [53][49].

ProSe UE App: This entity is responsible for building the application functionality. The ProSe UE App communicate and discover other ProSe UEs by means of the PC5 interface [50] .It is used by different services such as Public Safety or media application in order to get the requests and find buddies in proximity .

The ProSe Function is responsible of the different network actions to achieve ProSe requirements and it is used also to provide network services such as authorization, authentication, and data handling. The ProSe Function provides also the necessary charging and security functionality for usage of ProSe via the EPC.

The D2D communication architecture under LTE-A network defines 7 new interfaces to interconnect the new entities. These interfaces are called PC1, PC2, PC3, PC4, PC5, PC6 and PC7(Table 3).

The major roles of ProSe Fonction are given as follows:

- Provisioning (via the Direct Provisioning Function - DPF) Direct Provisioning Function (DPF) is used to provision the UE with necessary parameters in order use ProSe direct services. For direct communication used for Public Safety DPF is also used to provision the UE with parameters that are needed when the UE is not "served by E-UTRA". 
International Journal of Wireless \& Mobile Networks (IJWMN) Vol. 8, No. 1, February 2016

- Direct discovery management (via the Direct Discovery Name Management Function) :Direct Discovery Name Management Function is used for open Prose Direct Discovery to allocate and process the mapping of ProSe Applications IDs and ProSe Application Codes used in ProSe Direct Discovery. It uses ProSe related subscriber data stored in HSS for authorisation for each discovery request. It also provides the UE with the necessary security material in order to protect discovery messages transmitted over the air.

- EPC discovery (via the EPC-level Discovery ProSe Function ):EPC-level Discovery ProSe Function has a reference point towards the Application Server (PC2), towards other ProSe Functions (PC6), towards the HSS (PC4a) and the UE (PC3).

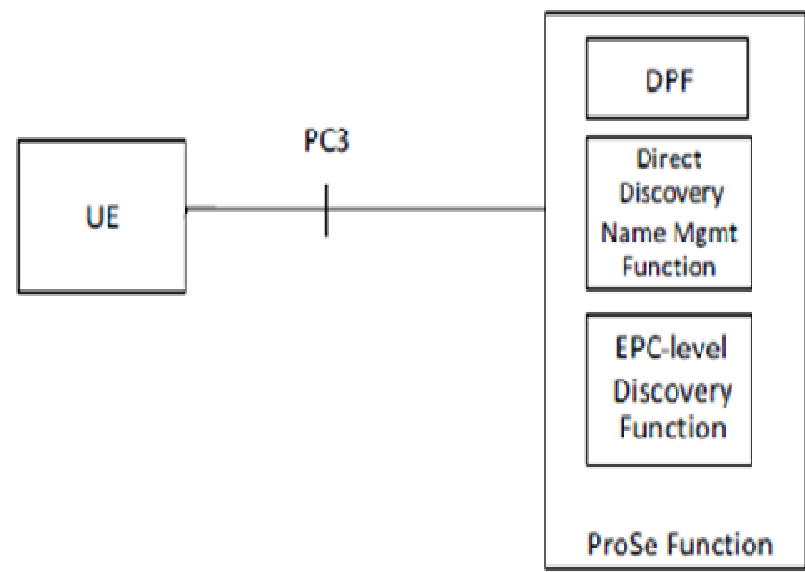

Figure 3. UE to ProSe Function Interface

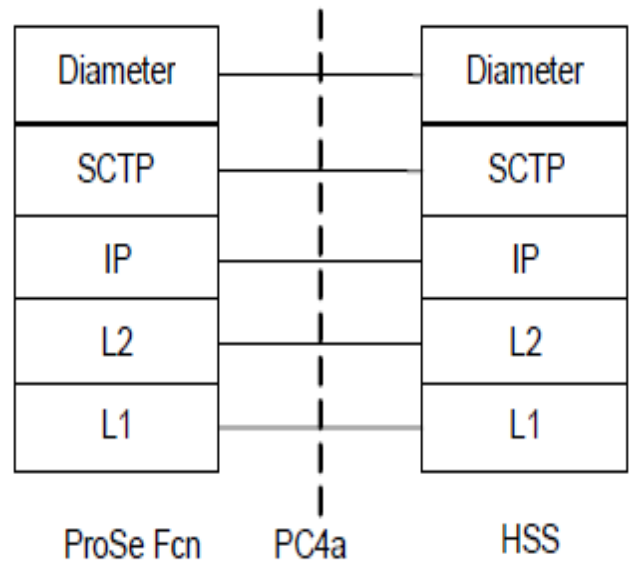

Figure 4. : Control Plane for PC4a Interface 
International Journal of Wireless \& Mobile Networks (IJWMN) Vol. 8, No. 1, February 2016

Table 3. Interfaces and Reference Points for D2D architecture under LTE-A Networks

Interface Description

PC1 It is the interface between the UE ProSe application and the ProSe Application Server. It is used to provide the level signalling requirements definition .

PC2 It is the interface between the ProSe Application Server and the ProSe Function. It is used to define the interaction between ProSe Application Server and ProSe functionality [47]. the ProSe Function use this interface to supports EPC-level discovery by the following functionalities.:

- Storage of a list of applications that are authorized to use EPC-level ProSe Discovery.

- Exchange of signalling with 3rd party Application Servers for application registration and identifier mapping.

The reference point between the UE and the ProSe Function (Figure.3) it is based on IP protocol and used for the ProSe Direct Discovery authorisation and to define the authorisation policy per PLMN for ProSe Direct Discovery and communication

PC3 between UE and ProSe Function.

PC4a The reference point between the HSS and ProSe Function .It is responsible to manage the subscription information and access authorisation for ProSE Direct Discovery and ProSE Direct Communication . More details on procedures, message parameters and protocol specifications of this interface are given in The [52] document. The PC4a interface is based on Diameter protocol. Over The PC4a interface, Diameter messages over the PC4a interface shall make use of SCTP IETF RFC 4960 (Figure.4).

PC5 . ProSe UEs are interconnected with each other by means of PC5 interface that is based on "ProSE protocol" that is used for handling ProSe Direct Discovery[51].

PC6 PC7 interface is used for the ProSe service and ProSe Direct Discovery requests authorization [49].

Interconnected the visited ProSe Function and the home ProSe Function .It is used to control ProSE service authorisation in the home ProSe Function.

\section{Conclusions}

Device-to-device (D2D) communications is seen as new paradigm that will be implemented in the next generations of mobile networks to provide high performance in cellular network, improving coverage, provide spectral efficiency, high data rates and offer new peer-to-peer services with QoS guarantees . 
International Journal of Wireless \& Mobile Networks (IJWMN) Vol. 8, No. 1, February 2016

In this paper, We present the different categories of D2D communication called Inband and Outband, we discuss related work and the different modes of each category .We also discussed the weaknesses and strength for each category.

Finally, we give a description of entities, interfaces, and protocols for the D2D communication architecture under LTE-A network based on LTE SAE architecture. We focus on the innovative architecture of D2D currently examined by 3GPP for integrating D2D communications in LTE-A networks.

In our future paper, we will use different statistical models and traffic models to study and analyse the different requirements to achieve high speed download, high capacity, and Quality of service guarantees for D2D communication. Furthermore, we will discuss challenges, solutions, and future directions for D2D communications in next generation 5G mobile networks.

\section{REFERENCES}

[1] E.SREE HARSHA1, T. TIRUPAL2 "LTE-Advanced Cellular Networks for D2D Communications" International Journal of Scientific Engineering and Technology Research Volume.03, IssueNo.18, August-2014.

[2] Arash Asadi ,Qing Wang, , and Vincenzo Mancuso," A Survey on Device-to-Device Communication in Cellular Networks" arXiv:1310.0720v6 [cs.GT] 29 Apr 2014.

[3] Doppler, K.; Rinne, M.P.; Janis, P.; Ribeiro, C.; Hugl, K "Device-to-Device Communications; Functional Prospects for LTE-Advanced Networks" ICC Workshops 2009.

[4] Xuemin Shen "Device-to-Device Communication in 5G Cellular Networks" IEEE Network March/April 2015 .

[5] Dimitris Tsolkas, Eirini Liotou, Nikos Passas and Lazaros Merakos "LTE-A Access, Core, and Protocol Architecture for D2D Communication "Springer International Publishing Switzerland 2014 .

[6] Zhijian Lin • Zhibin Gao • Lianfen Huang • Chi-Yuan Chen • Han-Chieh Chao "Hybrid Architecture Performance Analysis for Device-to-Device Communication in 5G Cellular Network" (C) Springer Science+Business Media New York 2015.

[7] K. Akkarajitsakul, P. Phunchongharn, E. Hossain, and V. K. Bhargava, "Mode selection for energyefficient D2D communications in LTEadvanced networks: A coalitional game approach," in Proceedings of IEEE ICCS, 2012, pp. 488-492.

[8] Chithra B Das "A Study on Device To Device Communication in Wireless Mobile Network" International Journal of Modern Communication Technologies \& Research (IJMCTR) ISSN: 23210850, Volume-3, Issue-3, March 2015.

[9] T. Peng, Q. Lu, H. Wang, S. Xu, and W. Wang, "Interference avoidance mechanisms in the hybrid cellular and device-to-device systems," in Proceedings of IEEE PIMRC, 2009, pp. 617-621.

[10] C.-H. Yu, K. Doppler, C. Ribeiro, and O. Tirkkonen, "Performance impact of fading interference to device-to-device communication underlaying cellular networks," in Proceedings of IEEE PIMRC, 2009, pp. 858-862.

[11] H. E. Elkotby, K. M. Elsayed, and M. H. Ismail, "Exploiting interference alignment for sum rate enhancement in D2D-enabled cellular networks," in Proceedings of IEEE WCNC, 2012, pp. 16241629

[12] A.Osseiran, K. Doppler, C. Ribeiro, M. Xiao, M. Skoglund, and J. Manssour, "Advances in device-todevice communications and Network coding for IMT-Advanced," ICT Mobile Summit, 2009

[13] C. Xu, L. Song, Z. Han, Q. Zhao, X. Wang, and B. Jiao, "Interference aware resource allocation for device-to-device communications as an underlay using sequential second price auction," in Proceedings of IEEE ICC, 2012, pp. 445-449.

[14] S. Xu, H. Wang, T. Chen, Q. Huang, and T. Peng, "Effective interference cancellation scheme for device-to-device communication underlaying cellular networks," in Proceedings of IEEE VTC-Fall, 2010, pp. 1-5.

[15] W. Xu, L. Liang, H. Zhang, S. Jin, J. C. Li, and M. Lei, "Performance enhanced transmission in device-to-device communications: Beamforming or interference cancellation?" in Proceedings of IEEE GLOBECOM, 2012, pp. 4296-4301. 
International Journal of Wireless \& Mobile Networks (IJWMN) Vol. 8, No. 1, February 2016

[16] B. Zhou, H. Hu, S.-Q. Huang, and H.-H. Chen, "Intracluster deviceto- device relay algorithm with optimal resource utilization," IEEE Transactions on Vehicular Technology, vol. 62, no. 5, pp. 23152326, Jun. 2013.

[17] X. Bao, U. Lee, I. Rimac, and R. R. Choudhury, "DataSpotting: offloading cellular traffic via managed device-to-device data transfer at data spots," ACM SIGMOBILE Mobile Computing and Communications Review, vol. 14, no. 3, pp. 37-39, 2010.

[18] X. Chen, L. Chen, M. Zeng, X. Zhang, and D. Yang, "Downlink resource allocation for device-todevice communication underlaying cellular networks," in Proceedings of IEEE PIMRC, 2012, pp. 232-237

[19] M. Ji, G. Caire, and A. F. Molisch, "Wireless device-to-device Caching networks: Basic principles and system performance,"arXiv preprintarXiv:1305.5216, 2013.

[20] H. Min, J. Lee, S. Park, and D. Hong, "Capacity enhancement of an interference limited area for device-to-device uplink underlaing cellular networks," IEEE Transactions on Wireles Communications, vol. 10, no. 12, pp. 3995-4000, December 2011.

[21] G. Fodor, E. Dahlman, G. Mildh, S. Parkvall, N. Reider, G. Mikls,and Z. Turnyi, "Design aspects of network assisted device-to- device communications," IEEE Communications Magazine, vol. 50, no. 3, pp.170-177, 2012.

[22] R. Zhang, X. Cheng, L. Yang, and B. Jiao, "Interference-aware Graph based resource sharing for device-to-device communications underlaying cellular networks," in Proceedings of IEEE WCNC, 2013, pp. 140-145.

[23] J. C. Li, M. Lei, and F. Gao, "Device-to-device (D2D) Communication in MU-MIMO cellular networks," in Proceedings of IEEE GLOBE COM,2012, pp. 3583-3587.

[24] N. Golrezaei, A. F. Molisch, and A. G. Dimakis, "Base-station assisted device-to-device communications for high-throughput wire less video networks," in Proceedings of IEEE ICC, 2012, pp. 7077-7081.

[25] Bin Guo, Shaohui Sun, Qiubin Gao "Interference Management for D2D Communications Underlying Cellular Networks at Cell Edge" ICWMC 2014.

[26] S. Shalmashi, G. Miao, and S. Ben Slimane, "Interference management for multiple device-to-device communications underlaying cellular networks," IEEE International Symposium on Personal Indoor and Mobile Radio Communications (PIMRC), 2013, pp. 223-227.

[27] J. Shin, J. P. Choi, and J.-W. Choi , "An autonomous interference avoidance scheme for D2D communications through frequency overhearing," International Conference on ICT Convergence (ICTC), 2013, pp. 1074-1075.

[28] H. Min, and J. Lee, "Capacity Enhancement Using an Interference Limited Area for Device-toDevice Uplink Underlaying Cellular Networks," IEEE Transactions on Wireless Communications, vol. 10, no. 12, Dec. 2011, pp. 3995-4000.

[29] K. Doppler, M Rinne, C. Wijiting, C. B. Ribeiro, and K. Hugl, "Device-to-Device Communication as an Underlay to LTE-Advanced Networks," Communications Magazine, IEEE, vol. 47, no. 12, Dec. 2009, pp 42-49. Copyright

[30] Ji Lianghai Klein, A. ; Kuruvatti, N. ; Schotten, H.D "System Capacity Optimization Algorithm for D2D Underlay Operation "Communications Workshops (ICC), 2014 IEEE.

[31] B. Zhou, H. Hu, S.-Q. Huang, and H.-H. Chen, "Intracluster deviceto-device relay algorithm with optimal resource utilization," IEEE Transactions on Vehicular Technology, vol. 62, no. 5, pp. 23152326, Jun. 2013.

[32] J. C. Li, M. Lei, and F. Gao, "Device-to-device (D2D) communication in MU-MIMO cellular networks," in Proceedings of IEEE GLOBECOM,2012, pp. 3583-3587.

[33] G. Fodor, E. Dahlman, G. Mildh, S. Parkvall, N. Reider, G. Mikls, and Z. Turnyi, "Design aspects of network assisted device-to-device communications," IEEE Communications Magazine, vol. 50, no. 3, pp. 170-177, 2012.

[34] W. ALliANCE, "Wi-Fi Peer-to-Peer (P2P) Specification v1. 1," WI-FI ALLIANCE SPECIFICATION, vol. 1, pp. 1-159, 2010

[35] Z. Alliance, “Zigbee specification,” Document 053474r06, Version, vol. 1, 2006.

[36] S. Bluetooth, "Bluetooth specification version 1.1," Available HTTP: http://www. bluetooth. com, 2001

[37] A. Asadi and V. Mancuso, "Energy efficient opportunistic uplink Packet forwarding in hybrid wireless networks," in Proceedings of the fourth international conference on Future energy systems, 2013. 
International Journal of Wireless \& Mobile Networks (IJWMN) Vol. 8, No. 1, February 2016

[38] On the compound impact of opportunistic scheduling and D2D communications in cellular networks, Accepted for publication in ACM MSWIM, 2013.

[39] C. Xu, L. Song, Z. Han, D. Li, and B. Jiao, "Resource allocation using a reverse iterative combinatorial auction for device-to-device underay cellular networks," in Proceedings of IEEE GLOBECOM, 2012, pp. 4542-4547.

[40] A. Asad , V.Mancuso" On the compound impact of opportunistic scheduling and D2D communications in cellular networks", Accepted for publication in ACM MSWIM, 2013.

[41] A. Asad , V.Mancuso" "WiFi Direct and LTE D2D in action," Accepted for publication in IEEE Wireless Days, 2013.

[42] N. Golrezaei, A. G. Dimakis, and A. F. Molisch, "Device-to-device collaboration through distributed storage," in Proceedings of IEEE GLOBECOM, 2012, pp. 2397-2402.

[43] A. Asadi and V. Mancuso, "Energy efficient opportunistic uplink packet forwarding in hybrid wireless networks," in Proceedings of the fourth international conference on Future energy systems, 2013, pp. 261-262.

[44] R1-132861. Final report of 3GPP TSG RAN WG1 \#73 v1.0.0, August 2013.

[45] 3GPP TR 22.803 v12.1.0, "Feasibility study for proximity services (ProSe)" 2013

[46] R1-132115, "Discussions on LTE device to device communication", ZTE, May 2013

[47] 3GPP TS 29.343 V12.1.0 (2014-12), "Proximity-services (ProSe) function to ProSe application server aspects (PC2)"; Stage 3 (Release 12).

[48] B.Raghothaman, E. Deng, R. Pragada, G. Sternberg, T. Deng, and K.Vanganuru, "Architecture and protocols for LTE-based device to device communication," in Proc. IEEE Int. Conf. Computing, Networking Communications, San Diego, CA, Jan. 2013, pp. 895-899.

[49] 3GPP TS 23.303 version 12.2.0 Release 12, Proximity-based services (ProSe); Stage.2. ETSI TS 123 303 V12.2.0. 2014-09.

[50] Athul Prasad, Andreas Kunz, Genadi Velev, Konstantinos Samdanis, and JaeSeung Song, Energy Efficient D2D Discovery for Proximity Services in 3GPP LTE-Advanced Networks. IEEE vehicular technology magazine ,December 2014.

[51] 3GPP TS 24.334: "Proximity-services (Prose) User Equipment (UE) to Proximity-services (ProSe) Function aspects (PC3); Stage 3".June2014

[52] 3GPP TS 29.344 V12.3.0 (2015-06), Proximity-services (ProSe) Function to Home Subscriber Server (HSS) aspects; Stage 3 (Release 12), June 2015.

[53] S. Mumtaz and J. Rodriguez (eds.), Smart Device to Smart Device Communication, DOI: 10.1007/978-3-319-04963-2_1,Springer International Publishing Switzerland 2014.

\section{Authors}

Mr. Margi Hicham ,He received the engineer degree in Telecommunication systems from The National Institute of Posts and Telecommunications (INPT) in Rabat, Morocco in 2011.He is currently working toward the Ph.D. degree at RITM Research Lab,ESTC ,Hassan II University ,Casablanca, Morocco. His research interests include Network Science, Wireless Ad hoc Networks and Sensor Networks. . His current research interests include cognitive radio systems, cooperative (relay) communications,D2D communications ,LTE-A and 5G wireless communications.

$\mathrm{Mr}$. Noreddine Abghour ,He is currently associate professor in the Faculty of Science of Hassan II University, Morocco. He received his Ph.D. degree from Institut National Polytechnique de Toulouse (France) in 2004. His research mainly deals with authorization schemes in distributed computing systems, and Currently, his research interests concern the problems related to the Mobile Communication Systems and Cloud Computing.
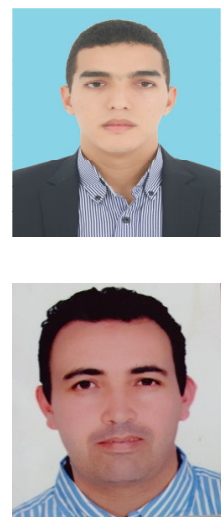

Mr. Mohammed Ouzzif, $\mathrm{He}$ is an associate Professor at the University Hassan II of Casablanca. He earned a PHD in the field of the specification and verification of distributed systems at the University Mohammed V in Rabat. Currently, his research interests concern the problems related to the wireless sensor networks and big data in the context of smart cities.

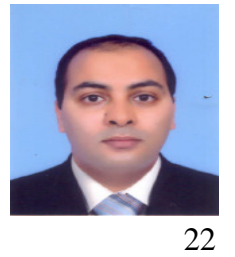

